\title{
A produção científica brasileira sobre Gestão Econômica em periódicos e eventos no período de 1989-2012
}

The Brazilian scientific production on Economic Management in journals and conferences in the period of 1989-2012

\author{
Sérgio Lemos Duarte \\ sergioufu@gmail.com \\ USP
}

Sara Isabel Melo Pereira saramelop@gmail.com USP

\author{
Sandro Vieira Soares \\ sandrovs@usp.br \\ USP
}

$\begin{gathered}\text { Juliana Ventura Amaral } \\ \text { juliana.ventura.amaral@usp.br }\end{gathered}$
USP

\author{
Carlos Alberto Pereira \\ cap@usp.br \\ USP
}

\begin{abstract}
Resumo: Os estudos sobre o sistema de Gestão Econômica começaram na década de 1970 como iniciativa do professor Armando Catelli. Após este período as pesquisas foram evoluindo em teses, dissertações, artigos em periódicos e eventos. O objetivo deste trabalho foi identificar as características dos artigos sobre Gestão Econômica em eventos e periódicos do período de 1989 a 2012. Os periódicos analisados estão ligados ou são mantidos pelos programas de pós graduação stricto sensu, online, em Controladoria e Contabilidade, e os anais analisados pertencem a eventos que são classificados como E1 e possuem alguma linha de interesse ou área temática em Contabilidade. Através da busca pelas palavras-chave "GECON", "Gestão Econômica" e "Resultado Econômico" no corpo do texto dos artigos, e de uma análise criteriosa dos achados, foram selecionados um total de 93 artigos (11 em periódicos e 82 em eventos). O estudo mostra que apenas 4 periódicos da amostra abrangem publicações da temática GECON e que, relativamente aos eventos analisados, existe uma forte concentração dos trabalhos no Congresso Brasileiro de Custos. Detectou-se ainda que o auge das publicações de GECON se verificou no período de 2001 a 2005, quando analisado o conjunto de eventos e periódicos. Relativamente às características dos artigos, constatou-se que os livros representam a principal fonte de referência dos autores e que, em média, os artigos publicados em eventos são elaborados por mais autores comparativamente com os artigos dos periódicos.
\end{abstract}

Palavras-chave: GECON. Resultado Econômico. Bibliometria.

Abstract: Studies on the Economic Management System began in 1970 as an initiative of Professor Armando Catelli. After this period, the research was evolving in theses, dissertations, journal articles, and conference papers. The objective of this article is to identify characteristics of conference papers and journal articles pertaining to Economic Management from 1989 to 2012. The journals that were analyzed are maintained by post graduate programs, are available online, and pertain to Controllership and Accounting. The annals that were analyzed are from E1 Conferences related to Accounting. By searching for the keywords GECON, Economic Management and Economic Profit in the text of the articles, and after careful analysis of the articles, a total of 93 articles were selected (11 from journals and 82 from conferences). This study shows that only four journals of the sample contained articles

R. Cont. Ufba, Salvador-Ba, v. 9, n. 1, p. 41 - 57, jan-mar 2015 
are related to GECON, and that of the conference annals analyzed, there is a strong concentration of work in the Brazilian Congress of Costs. Upon analyzing the set of conference annals and journals, it was also found that the height of publishing on the topic of GECON was from 2001 to 2005. Regarding the characteristics of the articles, it was found that the books represent a major source of reference for authors and that on average, the articles published in conference annals are authored by more authors when compared to the number of authors for journal articles.

Keywords: GECON. Economic result. Bibliometrics.

Artigo recebido em: 21/11/2013; Aceito em: 13/11/2014

\section{INTRODUÇÃO}

As pesquisas sobre o Modelo Gestão Econômica (GECON) tiveram início no Brasil na década de 1970 no âmbito do Programa de Pós-Graduação em Controladoria e Contabilidade da Faculdade de Economia, Administração e Contabilidade da Universidade de São Paulo PPGCC/FEA/USP, com o professor Armando Catelli. Desde então, o debate evoluiu e tornou o GECON um dos modelos gerenciais mais estudados no país: inúmeras teses, dissertações e tmonografias foram defendidas, livros foram escritos e artigos foram publicados. Alguns destes trabalhos visaram demonstrar a evolução do estudo do tema GECON, como os trabalhos de Passos et al (2010), Carvalho et al (2010), Ribeiro et al (2011), Cruz, Pereira e Espejo (2011) e Sales et al (2011).

Este trabalho vem no seguimento destas pesquisas, e busca responder à seguinte questão: Quais são as características das pesquisas sobre GECON publicadas em periódicos e eventos acadêmicos brasileiros entre os anos de 1989 e 2012? Para tal, este trabalho se desenvolve sob a forma de uma pesquisa bibliométrica, através de uma revisão de artigos que foram publicados em revistas ou em eventos científicos sobre o tema.

A justificativa desta pesquisa é ofertar aos interessados em GECON, sejam eles pesquisadores ou profissionais de mercado "extra-academia", um panorama acerca deste sistema de gestão, já que até ao momento nenhuma pesquisa teve o objetivo de fazer uma análise bibliométrica com o escopo deste trabalho. O GECON, quando foi criado na década de 1970 trouxe para o conjunto de técnicas gerencias a importância da análise econômica e financeira da empresa. Com o passar dos anos as pesquisas foram evoluindo ao passo que atualmente busca-se simplificar e customizar o método para aplicação nas diversas empresas e possibilitar análises pontuais de características de cada setor.

Os estudos bibliométricos são considerados relevantes para avaliar determinada área do conhecimento a fim de diagnosticar em que estado ela se encontra (SPLITTER; ROSA, 2012). Para Vanti (2002) as técnicas bibliométricas possuem como principais possibilidades de aplicação: (i) identificar as tendências e a expansão de uma área; (ii) prever tendências de publicação; (iii) prever a produtividade de autores individuais, organizações e países; (iv) identificar as revistas do núcleo de uma disciplina; (v) medir o crescimento de determinadas áreas e o surgimento de novos temas; e (vi) analisar a fonte de dados utilizada nos estudos. Os estudos bibliométricos podem ser realizados nas inúmeras áreas da Contabilidade (SOARES et al., 2012).

A contribuição inovadora deste trabalho consiste em: (1) identificar que o tema GECON foi alvo de pesquisas veiculadas em eventos científicos encontrando seu auge em 2002 quando foram veiculados 10 artigos nos eventos e em 2003 e 2004 quando houve a publicação de 2 artigos em cada ano no conjunto de revistas analisado; (2) identificar que o interesse por esse

R. Cont. Ufba, Salvador-Ba, v. 9, n. 1, p. 41 - 57, jan-mar 2015 
tema de pesquisa começou a decrescer a partir de 2005, conforme mostra a redução de publicações sobre o tema nos eventos e periódicos analisados; (3) identificar os autores que mais publicaram sobre o tema, nomeadamente Catelli, A., Parisi, C., Ponte, V. M. R., Pereira, C. A., Santos, E. S., vinculados principalmente ao programa PPGCC/USP e a UFC, e todos os artigos foram publicados no Brasil; (4) identificar que as principais revistas utilizadas pelos autores para veicular suas pesquisas sobre Gecon são a Revista de Contabilidade e Finanças (USP), Contabilidade Vista e Revista (UFMG), Revista Contemporânea de Contabilidade (UFSC) e Contextus - Revista Contemporânea de Economia e Gestão (UFC); (5) identificar que existe uma relação de orientação de dissertações de mestrado e teses de doutorado entre os principais autores.

A estrutura deste trabalho divide-se em: Introdução, onde o objetivo do trabalho é exposto; Revisão de Literatura, onde se apresenta o GECON; Metodologia, onde se faz a classificação metodológica da pesquisa e se descreve a sua trajetória; Resultados, onde se apresentam os principais achados da pesquisa; e Conclusões, onde se discute acerca do atual estado da arte da pesquisa sobre GECON, as suas tendências e perspectivas futuras.

\section{O MODELO DE GESTÃO ECONÔMICA}

A crescente competitividade do mercado, principalmente na segunda metade do século XX, intensificou a necessidade das empresas buscarem instrumentos de gestão e de métodos de operação mais eficientes (PEREIRA, 2011). Foi neste contexto que o professor Armando Catelli, no final da década de 1970, idealizou o modelo de gestão econômica com o objetivo de suprir esta nova necessidade, que os mercados trazem para as empresas, e auxiliar as empresas na tomada de decisão.

O GECON é um sistema de gestão baseado em resultados econômicos com o objetivo de auxiliar os gestores na tomada de decisão e, segundo Guerreiro, Catelli e Dornelles (1997), refere-se a um modelo gerencial para administração por resultados econômicos, que visa a eficácia empresarial.

Desta forma, o processo de gestão empresarial passa por novos desafios e os gestores passam a trabalhar com novos modelos de decisão. Segundo Catelli et al. (2010a), as empresas necessitam de uma abordagem holística para cumprir com seus desafios e, assim, devem implementar métodos e técnicas adequadas nos subsistemas de uma empresa, como na organização, no modelo de gestão, no sistema de gestão e de informação, nos processos operacionais, e, sobretudo, promover a competência das pessoas para que alcancem os objetivos propostos pela companhia.

\subsection{Resultado Econômico e Eficácia Empresarial}

O cumprimento da missão de uma empresa constitui-se em seu objetivo maior. Assim, uma empresa pode ser considerada eficaz quando consegue cumprir com a sua missão e ter continuidade (PEREIRA, 2011).

No entanto, medir a eficácia empresarial por meio do cumprimento de sua missão não é tarefa fácil. Para tanto, o conceito de eficácia deve ser operacionalizado de forma que se tenham indicadores mensuráveis e de uso prático. Pereira (2011) assegura que a eficácia empresarial, por se tratar de um conceito multidimensional, envolve fatores como a produtividade, a eficiência, a sobrevivência, o desenvolvimento, a adaptabilidade e a satisfação nas diferentes dimensões de desempenho de uma empresa. 
Neste contexto, Catelli et al. (2010b) destacam que a eficácia econômica de qualquer instituição decorre do grau de satisfação que proporciona às diversas entidades com as quais se relaciona, tais como: clientes, acionistas, governo, pessoal, sindicatos, fornecedores etc.

A flexibilidade e a capacidade de adaptação da empresa ao seu ambiente refletem-se diretamente em seus resultados econômicos, que espelham e determinam suas condições de continuidade num ambiente essencialmente dinâmico.

Desta forma, o resultado econômico, corretamente mensurado, apresenta-se como a melhor medida da eficácia de uma organização, visto que, por meio da exploração de suas atividades, corresponde ao valor adicionado ao seu patrimônio em um dado período.

\subsection{Estrutura conceitual do sistema de informações de gestão econômica}

Quatro modelos atuam de forma integrada e atuam como alicerce à gestão econômica:

- Modelo de gestão: Trata-se do conjunto de princípios, crenças e valores que definem a forma de gestão da empresa e que influenciam as diversas atividades da organização e o processo de tomada de decisão. Estes princípios estão de acordo com a missão da empresa e funcionam como um elemento direcionador para que a empresa tenha um comportamento favorável ao cumprimento da sua missão.

- Modelo de decisão: Engloba o processo decisório, ou seja, como os gestores devem proceder na sua tomada de decisão, levando em conta o processo de gestão, as diversas atividades e eventos realizados ou planejados pela empresa. Este modelo é essencial para a construção de um sistema de informação que atenda às necessidades dos gestores.

- Modelo de mensuração: Indica o modo de mensuração dos eventos, transações e atividades sobre os quais os gestores tomam as suas decisões,

- Modelo de informação: envolve o conjunto de características que devem ser processadas pelo sistema de informação. Guerreiro (1989) cita as características das informações que devem compor o modelo de informação do GECON: adequação à decisão, oportunidade, precisão, relevância e objetividade; valor econômico - seu benefício deve ser superior ao custo de geração da informação; Relatividade - a informação sobre um dado deve possuir relação com outro de mesma natureza; exceção - a informação deve comunicar as ocorrências que não estavam preestabelecidas; entendimento - as informações devem ser facilmente compreensíveis pelo usuário; acionabilidade - a informação deve ser dirigida aos gestores que efetivamente influenciam a ação ou possuem o poder de decisão; motivação - a informação deve motivar os tomadores de decisão a otimizarem o resultado global; consistência e integração; uniformidade de critérios; indicar as causas das variações entre o resultado planejado e ocorrido; volume - a quantidade deve ser compatível no auxílio à tomada de decisão; seletividade - as informações devem ser previamente selecionadas, visando atender às necessidades específicas dos gestores; generalidade - as informações contidas no sistema de informação devem atender a um grande número de usuários, sem que seja necessária nenhuma modificação fundamental no sistema; flexibilidade; e tempo de resposta - as informações solicitadas devem ser atendidas rapidamente.

\section{PESQUISAS ANTERIORES SOBRE O TEMA}

Alguns trabalhos visaram tratar sobre a amplitude das pesquisas de Gestão Econômica em periódicos e eventos no Brasil, cada qual com um escopo distinto: Carvalho et al (2010), Passos et al (2010), Ribeiro et al (2011), Cruz, Pereira e Espejo (2011) e Sales et al (2011). As fontes utilizadas para pesquisa em cada trabalho estão demonstradas no quadro 1.

R. Cont. Ufba, Salvador-Ba, v. 9, n. 1, p. 41 - 57, jan-mar 2015 
Quadro 1: Fonte de dados pesquisadas pelos autores.

\begin{tabular}{|c|c|}
\hline Artigo & Fontes analisadas por evento/periódico e período \\
\hline Carvalho et al (2010) & $\begin{array}{l}\text { Eventos: Anpcont (2007-2009), EnAnpad (1997-2009), USP (2001-2010) } \\
\text { Periódicos: CVR (1989-2010), RC\&F (1989-2010), RCC (2004-2010), RCCMCC } \\
\text { (2003-2010), RCO (2007-2010), REPEC (2007-2010), PC (2004-2010), RIC } \\
(2007-2010), \text { RUC (2005-2010) }\end{array}$ \\
\hline Passos et al (2010) & $\begin{array}{l}\text { Periódicos: RAUSP (1993-2008), RAE (1993-2008), PPE (1993-2008), C\&F } \\
\text { (1993-2008), CVR (1993-2008), READ (1995-2008), RN (1996-2008), RCCMCC } \\
\text { (2002-2008), RAC (1997-2008), RCA (1999-2008), RBGN (2004-2008), GP } \\
\text { (2002-2006), RAM (2000-2008), FACES (2002-2008), RAE-e (2002-2008), } \\
\text { Alcance (2005-2008), RCC (2004-2007), BASE (2004-2008), BBR (2004-2008), } \\
\text { RAI (2004-2008), RUC (2005-2008), G.ORG (2004-2008), RBF (2005-2008) }\end{array}$ \\
\hline Ribeiro et al (2011) & Evento: Congresso Brasileiro de Custos (2004-2010) \\
\hline $\begin{array}{c}\text { Cruz, Pereira e Espejo } \\
\text { (2011) }\end{array}$ & Evento: Congresso Brasileiro de Custos (2004-2009) \\
\hline Sales et al (2011) & $\begin{array}{l}\text { Eventos: Anpcont (2007-2009), EnAnpad (1997-2009), USP (2001-2010) } \\
\text { Periódicos: CVR (1989-2010), RC\&F (1989-2010), RCC (2004-2010), RCCMCC } \\
\text { (2003-2010), RCO (2007-2010), REPEC (2007-2010), PC (2004-2010), RIC } \\
(2007-2010), \text { RUC (2005-2010) }\end{array}$ \\
\hline
\end{tabular}

Fonte: Dados da Pesquisa.

O primeiro deles é o estudo dos autores Carvalho et al (2010) que realizaram uma pesquisa cujo objetivo incidiu sobre a análise das metodologias e técnicas de pesquisa dos trabalhos de GECON. Realizaram a busca pelos artigos nos periódicos e eventos relacionados no quadro 1, pelas palavras-chave: "Gecon, Sistema de Gestão Econômica e Gestão Econômica", encontrando 18 trabalhos para análise. As principais conclusões obtidas pelos autores revelam que, na sua maioria, os artigos não explicitam o problema de pesquisa e metodologia de elaboração, atribuindo importância apenas aos objetivos do estudo. Revelam também que os estudos são essencialmente descritivos, bibliográficos e/ou qualitativos e que são, geralmente, apresentados em congressos e outros eventos.

Já o estudo de Passos et al (2010) analisou vinte e quatro periódicos, tendo sido utilizado como método de pesquisa a análise do título, resumo ou palavra-chave. Desta forma, os autores conseguiram identificar, nesta população, 12 artigos relativos ao tema GECON, o que levou a que chegassem à conclusão que existiu uma baixa inserção do referido tema $(0,26 \%)$ no total de artigos publicados nos referidos periódicos.

Posteriormente Ribeiro et al (2011) no seu estudo bibliométrico sobre controladoria demonstrou que a obra "Controladoria: Uma abordagem da gestão econômica" do professor Catelli, é a mais citada nas publicações em controladoria do Congresso Brasileiro de Custos (CBC) de 2004 a 2010, sendo referenciado em 49 trabalhos no total de 215.

A pesquisa de Cruz, Pereira e Espejo (2011) revelara que o tema apresenta sinais de desenvolvimento nas publicações apresentadas no CBC. Para obterem esta conclusão os autores fizeram uma análise de conteúdo em 27 artigos, verificando que estes sinalizavam que o desenvolvimento de pesquisas sobre Gestão Econômica, com apoio em frameworks alternativos, ainda é bastante tímida no universo analisado.

Por fim Sales et al (2011), no artigo intitulado "22 anos de pesquisas contábeis sobre GECON" estão na mesma linha que as dos restantes autores, revelando que existem poucas publicações sobre o tema, totalizando apenas 17 publicações nos 22 anos de análise.

\section{METODOLOGIA}

R. Cont. Ufba, Salvador-Ba, v. 9, n. 1, p. 41 - 57, jan-mar 2015 
A metodologia desta pesquisa divide-se em duas seções: a classificação metodológica e a trajetória metodológica.

\subsection{Classificação metodológica}

A classificação desta pesquisa pode ser feita sob quatro perspectivas distintas: quanto aos objetivos, quanto a abordagem dos dados, quanto a lógica interna e quanto ao procedimento técnico.

Quanto à abordagem dos dados analisados, que são os artigos de periódicos e papers de eventos, esta pesquisa é classificada como quali-quantitativa. Esta classificação se justifica dado que se utiliza um foco em características qualitativas da pesquisa, mas também se utiliza de estatística descritiva para sintetizar as informações encontradas.

A amostra escolhida é classificada como intencional e não-probabilística. No entanto, a amostra analisada foi escolhida com base em critérios expostos por Eloy Jr., Soares e Casagrande (2013) e atualizados com dados do Qualis da Capes (2012).

Por fim, o procedimento técnico desta pesquisa classifica-se como um estudo bibliométrico. Tais estudos são realizados com o objetivo de introduzir o pesquisador junto a determinado tema e ainda permitir que se verifiquem as características ambientais da pesquisa sobre um determinado assunto. Isso possibilita ao autor, quando de posse dos trabalhos mais relevantes e atuais sobre o tema, ter uma visão do chamado "estado da arte" de determinado tema.

\subsection{Trajetória metodológica}

A escolha da amostra de revistas e eventos científicos analisados foi intencional e limitada pela acessibilidade. A quase totalidade das revistas brasileiras sobre contabilidade na área de Administração, Ciências Contábeis e Turismo não está indexada em grandes bases de dados internacionais como ISI, Scopus, Emerald ou Ebsco e também não possui fator de impacto como o JCR ou o H Scopus (com exceção da Revista Brasileira de Gestão de Negócios RBGN). Logo, estudos bibliométricos sobre determinados temas de Contabilidade no Brasil, baseados nestas bases de dados são improdutivos e não refletem a realidade da pesquisa contábil no país.

No entanto, no Brasil, a Coordenação de Aperfeiçoamento de Pessoal de Nível Superior CAPES - utiliza um modelo próprio de avaliação dos periódicos para os quais, cada área de conhecimento atribui critérios e pontuação correspondente, e assim, a Capes pode avaliar a qualidade da produção dos professores, pesquisadores e discentes dos programas de pósgraduação stricto sensu por ela regulados de acordo com a pontuação destes periódicos utilizados para escoar a produção de artigos.

A área de Administração, Ciências Contábeis e Turismo, por sua vez estabelece os critérios que são aplicados aos periódicos de Contabilidade e a comissão de representantes da área, torna a revisar a pontuação atribuída a cada periódico trienalmente com base no desempenho do periódico.

O estabelecimento dos critérios para classificação dos periódicos é considerado um avanço para a área dado que ele permite que os periódicos tracem estratégias para buscar melhores classificações no Qualis e também para o surgimento de novos periódicos. Isso porque, segundo o estudo de Murcia, Rosa e Borba (2013) para que todos os docentes de programas de pós-graduação stricto sensu em Contabilidade atingissem a pontuação em artigos para que o programa seja considerado muito bom (150 pontos no triênio) seria necessário que os 56 periódicos analisados publicassem um total de 37.650 pontos enquanto que estes publicaram, no triênio 2007-2009, apenas 23.490.

R. Cont. Ufba, Salvador-Ba, v. 9, n. 1, p. 41 - 57, jan-mar 2015 
Contudo, a identificação de 56 periódicos em Contabilidade pelo estudo de Murcia, Rosa e Borba (2013) foi realizada com base no seguinte critério: dos 837 periódicos da área de Administração, Ciências Contábeis e Turismo foram deduzidos 293 periódicos internacionais, 67 periódicos que não possuem site, 17 periódicos descontinuados, 50 periódicos que possuíam site, mas sem foco/escopo, 350 periódicos que não possuíam as palavras-chave selecionadas, 1 periódico que possuía as palavras-chave, mas não era da área contábil e 3 periódicos duplicados na lista por possuírem versão online e impressa. As palavras-chave utilizadas pelos autores foram contabilidade, ciências contábeis, contábil, controladoria, finanças e auditoria. No entanto os autores dividiram esses 56 periódicos em dois grupos: 15 revistas da área de Contabilidade e 41 revistas não somente da área da contabilidade.

Por sua vez, a pesquisa de Eloy Jr., Soares e Casagrande (2013) utilizou outro critério para estipular a amostra de revistas da área de Contabilidade que julgaram representativas da área: as revistas ligadas ou mantidas por programas de pós-graduação stricto sensu, online, em Contabilidade, ofertados no Brasil que são:

Quadro 2: Revistas analisadas, por IES, por UF e ano primeira edição online.

\begin{tabular}{|c|c|c|c|}
\hline Revista & IES & $\mathbf{U F}$ & Ano \\
\hline Contabilidade Vista e Revista & UFMG & MG & 1989 \\
\hline Revista Contabilidade e Finanças & USP & SP & 1989 \\
\hline Contabilidade, Gestão e Governança & UNB & DF & 1998 \\
\hline Contextus - Revista Contemporânea de Economia e Gestão & UFC & $\mathrm{CE}$ & 2003 \\
\hline Revista de Contabilidade do Mestrado em Ciências Contábeis da UERJ & UERJ & RJ & 2003 \\
\hline Brazilian Business Review & FUCAPE & ES & 2004 \\
\hline Base - Revista de Administração e Contabilidade da Unisinos & UNISINOS & $\mathrm{RS}$ & 2004 \\
\hline Revista Brasileira de Gestão de Negócios & UNIFECAP & SP & 2004 \\
\hline Revista Contemporânea de Contabilidade & UFSC & $\mathrm{SC}$ & 2004 \\
\hline Revista Universo Contábil & FURB & $\mathrm{SC}$ & 2005 \\
\hline Sociedade, Contabilidade e Gestão & UFRJ & RJ & 2006 \\
\hline Revista de Informação Contábil & UFPE & $\mathrm{PE}$ & 2007 \\
\hline Revista de Contabilidade da UFBA & UFBA & BA & 2007 \\
\hline Revista Contabilidade e Organizações & USP/RP & SP & 2007 \\
\hline Revista Contabilidade e Controladoria & UFPR & PR & 2009 \\
\hline
\end{tabular}

Fonte: Eloy Jr., Soares e Casagrande (2013).

A pesquisa de Eloy Jr., Soares e Casagrande (2013), no entanto não tratou apenas dos artigos em periódicos. Os autores julgaram oportuno estender a sua análise aos anais de eventos dado que estes, tradicionalmente, veiculam um número muito maior de relatos de pesquisa do que os periódicos, tanto que em seu estudo, os autores identificaram que os 15 periódicos analisados veicularam e mantém disponíveis online, entre 1989 e 2011, um total de 2.395 artigos enquanto que em cinco eventos considerados na pesquisa (CBC, EnEPQ, EnANPAD, AnpCont e USP) veicularam e mantém online, entre 1994 e 2011, um total de 14.473 artigos. Ao encontro deste resultado, embora de forma ainda mais acentuada, a pesquisa de Cruz et al. (2011) evidenciou que apenas $15 \%$ dos artigos publicados no Congresso USP de Controladoria e Contabilidade entre 2001 e 2010 tornam-se publicações definitivas.

O Documento de Área 2009, da área de Administração, Ciências Contábeis e Turismo, elenca uma lista de 106 eventos classificados nos estratos E1 (45) e E2 (61). Da mesma forma, nem todos os eventos são da área, e tampouco todos os eventos da área incluem a Contabilidade como escopo de forma que Eloy Jr., Soares e Casagrande (2013) decidiram por utilizar dois critérios para seleção da amostra: $1^{\circ}$ - Evento classificados como E1, que é o nível superior ao E2 e $2^{\circ}$ - Eventos que possuíam alguma linha de interesse ou área temática em Contabilidade e assim os eventos selecionados para ter seus anais examinados foram:

Quadro 3: Eventos por Instituição Organizadora e ano da primeira edição a ser consultada.

R. Cont. Ufba, Salvador-Ba, v. 9, n. 1, p. 41 - 57, jan-mar 2015 


\begin{tabular}{|c|c|c|}
\hline Evento & Instituição & Ano \\
\hline CBC - Congresso Brasileiro de Custos & ABC & 1994 \\
\hline $\begin{array}{c}\text { AnAministração } \\
\text { Adminion-Graduação e Pesquisa em }\end{array}$ & ANPAD & 1997 \\
\hline $\begin{array}{c}\text { Congresso USP de Controladoria e Contabilidade } \\
\text { USP }\end{array}$ & 2001 \\
\hline EnEPQ - Encontro de Ensino e Pesquisa em Administração e Contabilidade & ANPAD & 2007 \\
\hline $\begin{array}{c}\text { ANPCONT - Encontro da Associação dos Programas de Pós-Graduação em Ciências } \\
\text { Contábeis }\end{array}$ & ANPCONT & 2007 \\
\hline
\end{tabular}

Fonte: Dados da pesquisa.

Por fim, depois de selecionados os periódicos e eventos que compunham a amostra, procedeu -se à identificação dos artigos que tratam de GECON. Para isso, fez-se uma busca pelas palavras-chave "GECON", "Gestão Econômica" e "Resultado Econômico" no corpo do texto. Todos os artigos que possuíam as palavras-chave, mas que não tratavam do assunto (pela leitura de seu conteúdo) foram eliminados da análise.

Em relação aos periódicos analisados, a pesquisa abrange os artigos de todas as publicações desde os anos de origem dos periódicos até 2012, exceto no caso do periódico Base - Revista de Administração e Contabilidade da Unisinos, que devido à indisponibilidade dos artigos para consulta, apenas foram analisados os anos de 2008 a 2012.

No caso dos eventos analisados, esta pesquisa abarca todos os anos em que os eventos ocorreram e cujos anais encontram-se disponíveis online até 2012.

No decorrer da pesquisa foi encontrado, no periódico RIC- Revista da Informação Contábil, Vol. 2, No 3 (2008), o artigo "O Resultado Econômico na Gestão Pública: A mensuração de custo e resultado" dos autores Ely Corbari e Joel de Jesus Macedo, que reúne as características requeridas para fazer parte do estudo. No entanto, este artigo não se encontra disponível para consulta, pelo que não integra os resultados.

\section{ANÁLISE DE RESULTADOS}

O objetivo desta seção é apresentar os resultados alcançados na análise bibliométrica dos artigos coletados, nos periódicos e eventos referidos na seção anterior. Desta forma, esta seção iniciar-se-á com a apresentação dos resultados mais relevantes da pesquisa, para que, posteriormente, possam ser formuladas as devidas conclusões do estudo.

A Tabela 1 demonstra o total de artigos publicados nos periódicos que fazem parte da amostra deste estudo e o número de artigos que abordam a temática GECON.

Tabela 1: Frequência dos artigos sobre GECON nos periódicos analisados.

\begin{tabular}{|c|c|c|c|}
\hline Periódico & Artigos & GECON & $(\%)$ \\
\hline Contextus - Revista Contemporânea de Economia e Gestão & 145 & 1 & $0.69 \%$ \\
\hline Brazilian Business Review & 195 & 0 & $0.00 \%$ \\
\hline Contabilidade Vista e Revista & 458 & 4 & $0.87 \%$ \\
\hline Revista de Informação Contábil & 173 & 0 & $0.00 \%$ \\
\hline Sociedade, Contabilidade e Gestão & 167 & 0 & $0.00 \%$ \\
\hline Revista de Contabilidade do Mestrado em Ciências Contábeis da UERJ & 183 & 0 & $0.00 \%$ \\
\hline Base - Revista de Administração e Contabilidade da Unisinos & 116 & 0 & $0.00 \%$ \\
\hline Revista Universo Contábil & 317 & 0 & $0.00 \%$ \\
\hline Revista Brasileira de Gestão de Negócios & 241 & 0 & $0.00 \%$ \\
\hline Revista de Contabilidade da UFBA & 120 & 0 & $0.00 \%$ \\
\hline Revista Contabilidade e Controladoria & 110 & 0 & $0.00 \%$ \\
\hline Revista Contemporânea de Contabilidade & 165 & 2 & $1.21 \%$ \\
\hline Contabilidade, Gestão e Governança & 260 & 0 & $0.00 \%$ \\
\hline Revista Contabilidade e Finanças & 417 & 4 & $0.96 \%$ \\
\hline Revista Contabilidade e Organizações & 178 & 0 & $0.00 \%$ \\
\hline
\end{tabular}

R. Cont. Ufba, Salvador-Ba, v. 9, n. 1, p. 41 - 57, jan-mar 2015 
Fonte: Dados da Pesquisa.

Foram analisados quinze periódicos, dos quais apenas 4 tinham publicações com o tema GECON, sendo que os que apresentaram um maior número de publicações foram a Revista Contabilidade Vista e Revista e a Revista Contabilidade e Finanças.

$\mathrm{Na}$ Tabela 2 são apresentados o total de artigos que foram apresentados em eventos e os que trataram do tema específico GECON.

Tabela 2: Frequência dos artigos de GECON nos Eventos.

\begin{tabular}{|c|c|c|c|}
\hline Evento & $\mathrm{N}^{\circ}$ de artigos & Artigos GECON & $(\%)$ \\
\hline CBC & 3241 & 64 & $1.97 \%$ \\
\hline EnEPQ & 317 & 0 & $0,00 \%$ \\
\hline EnANPAD & 10577 & 7 & $0.07 \%$ \\
\hline AnpCont & 472 & 0 & $0.00 \%$ \\
\hline USP & 1419 & 11 & $0.78 \%$ \\
\hline Total & 16026 & 82 & $0.51 \%$ \\
\hline
\end{tabular}

Nos eventos houve uma maior frequência de artigos do tema investigado, num total de 82, dos quais o Congresso Brasileiro de Custos representa 78\%. É importante salientar a diferença entre o número de artigos em eventos em relação aos periódicos. Isto porque os artigos sobre GECON que estão sendo apresentados nos eventos não estão sendo publicados nos periódicos mantidos pelos programas de pós-graduação em Contabilidade.

A Tabela 3 demonstra o número de artigos de eventos e periódicos publicados por ano, desde 1995 a 2012.

Tabela 3: Total de Artigos de GECON em Eventos e Periódicos por Ano.

\begin{tabular}{|c|c|c|c|c|c|c|c|c|c|c|}
\hline \multirow[b]{2}{*}{ Ano } & \multicolumn{4}{|c|}{ Eventos } & \multicolumn{5}{|c|}{ Periódicos* } & \multirow{2}{*}{$\begin{array}{l}\text { Total } \\
\text { Geral }\end{array}$} \\
\hline & $\mathrm{CBC}$ & $\begin{array}{l}\text { Congr. } \\
\text { USP }\end{array}$ & EnANPAD & Total & CVR & Contextus & $\mathrm{RCF}$ & $\mathrm{RCC}$ & Total & \\
\hline 1995 & 2 & & & 2 & & & & & & 2 \\
\hline 1996 & 2 & & & 2 & & & & & & 2 \\
\hline 1997 & 4 & & & 4 & & & & & & 4 \\
\hline 1998 & 7 & & & 7 & & & & & & 7 \\
\hline 1999 & 8 & & & 8 & & & & & & 8 \\
\hline 2000 & 3 & & 1 & 4 & & & & & & 4 \\
\hline 2001 & 1 & 2 & 2 & 5 & 1 & & 1 & & 2 & 7 \\
\hline 2002 & 7 & 2 & 1 & 10 & & & 1 & & 1 & 11 \\
\hline 2003 & 5 & & 1 & 6 & & & 2 & & 2 & 8 \\
\hline 2004 & 7 & & 1 & 8 & & 1 & & 1 & 2 & 10 \\
\hline 2005 & 4 & 3 & & 7 & & & & & & 7 \\
\hline 2006 & 2 & 2 & 1 & 5 & 1 & & & & 1 & 6 \\
\hline 2007 & 3 & 1 & & 4 & & & & 1 & 1 & 5 \\
\hline 2008 & 2 & & & 2 & & & & & & 2 \\
\hline 2009 & 3 & & & 3 & & & & & & 3 \\
\hline 2010 & 2 & 1 & & 3 & & & & & & 3 \\
\hline 2011 & 2 & & & 2 & 1 & & & & 1 & 3 \\
\hline 2012 & & & & 0 & 1 & & & & 1 & 1 \\
\hline Total & 64 & 11 & 7 & 82 & 4 & 1 & 4 & 2 & 11 & 93 \\
\hline
\end{tabular}

Por meio da análise da Tabela 3, verifica-se que os anos de 2001 a 2005 apresentaram o maior número de artigos publicados quando analisado o conjunto de eventos e periódicos. Antes 
disto, nota-se que havia um crescimento nas pesquisas, e após 2005 começa um decréscimo nas publicações. Na pesquisa de Carvalho et al. (2010) havia uma concentração nos anos de 2003-2006, o que pode ser explicado pelas duas pesquisas terem se baseado em grupos de fontes de artigos diferente, embora tenham encontrado algum período de concentração de publicações em comum (2003-2005).

A Tabela 4 trata dos tipos de referências utilizadas nos diversos artigos analisados. Para tal, as referências foram analisadas e classificadas segundo o tipo de fonte.

Tabela 4: Tipo de Referência Utilizada (\% do Total das Referências)

\begin{tabular}{|c|c|c|c|c|}
\hline \multirow{2}{*}{ Referência } & \multicolumn{2}{|c|}{ Eventos } & \multicolumn{2}{|c|}{ Periódicos } \\
\hline & Quantidade & $(\%)$ & Quantidade & $(\%)$ \\
\hline Dissertações & 105 & $7,28 \%$ & 21 & $7,81 \%$ \\
\hline Monografias/TCC & 7 & $0,49 \%$ & 0 & $0,00 \%$ \\
\hline Livros & 799 & $55,37 \%$ & 113 & $42,01 \%$ \\
\hline Teses & 87 & $6,03 \%$ & 27 & $10,04 \%$ \\
\hline Artigos - periódicos & 197 & $13,65 \%$ & 47 & $17,47 \%$ \\
\hline Artigos - congressos & 109 & $7,55 \%$ & 11 & $4,09 \%$ \\
\hline Sites & 45 & $3,12 \%$ & 8 & $2,97 \%$ \\
\hline Legislação & 24 & $1,66 \%$ & 5 & $1,86 \%$ \\
\hline Outros & 70 & $4,85 \%$ & 37 & $13,75 \%$ \\
\hline (a) Total & 1443 & $100,00 \%$ & 269 & $100,00 \%$ \\
\hline (b) Total de artigos & 82 & - & 11 & - \\
\hline Média de referências $(\mathbf{a} / \mathbf{b})$ & 17,5 & - & 24,4 & - \\
\hline
\end{tabular}

Pela análise da tabela conclui-se que o tipo de fonte mais utilizado nas pesquisas foram os livros, que nos eventos representam $55,3 \%$ do total de referências e nos periódicos $42 \%$. Os artigos publicados nos periódicos sobre GECON referenciam mais informações contidas em artigos de outros periódicos, num percentual de 17,47\%, em relação aos eventos que utilizam $13,65 \%$. Verifica-se também que os artigos em periódicos não referenciam monografias e TCCs.

$\mathrm{Na}$ pesquisa de Ribeiro et al (2011) foi analisada as principais fontes consultadas e identificou-se que 58,19\% das fontes foram livros nacionais e internacionais. Esse índice se aproxima do encontrado por esta pesquisa quando se analisa apenas os periódicos $(55,37 \%)$ mas se distancia do índice encontrado para periódicos $(42,01 \%)$.

Tabela 5: Quantidade de autores por artigo em eventos e periódicos

\begin{tabular}{|c|c|c|c|c|}
\hline \multirow{2}{*}{ Número de autores } & \multicolumn{2}{|c|}{ Eventos } & \multicolumn{2}{|c|}{ Periódicos } \\
\hline & Quantidade & $(\%)$ & Quantidade & $(\%)$ \\
\hline Um autor & 20 & $24,39 \%$ & 3 & $27,27 \%$ \\
\hline Dois autores & 24 & $29,27 \%$ & 4 & $36,36 \%$ \\
\hline Três autores & 16 & $19,51 \%$ & 2 & $18,18 \%$ \\
\hline Quatro autores & 16 & $19,51 \%$ & 2 & $18,18 \%$ \\
\hline Cinco autores & 5 & $6,10 \%$ & 0 & $0,00 \%$ \\
\hline Seis autores & 1 & $1,22 \%$ & 0 & $0,00 \%$ \\
\hline Total & 82 & $100,00 \%$ & 11 & $100,00 \%$ \\
\hline Média de autores por artigo & 2,57 & - & 2,27 & - \\
\hline
\end{tabular}

Como mostra a Tabela 5 pode-se verificar que a grande maioria dos trabalhos é escrita em coautorias, correspondendo a 75,61\% em eventos e 72,73\% em periódicos. Em média, os artigos em eventos têm mais autores do que os artigos em periódicos, sendo que em eventos existem trabalhos com 5 e 6 autores, o que não ocorreu com os artigos de periódicos. Uma possível explicação para isso é a não aceitação, por parte dos periódicos, de artigos com mais de 4 autores.

R. Cont. Ufba, Salvador-Ba, v. 9, n. 1, p. 41 - 57, jan-mar 2015 
Na pesquisa de Cruz, Pereira e Espejo (2010) 88,89\% dos artigos foram escritos por mais de um autor, sendo que $81,49 \%$ tiveram entre 2 e 4 autores. Na pesquisa de Ribeiro et al (2011) $92,94 \%$ dos artigos foram escritos por mais de um autor, sendo que 84,22\% foram escritos por grupos de 2 a 4 autores. Ou seja, os achados desta pesquisa são corroborados por Cruz, Pereira e Espejo (2010) e Ribeiro et al (2011).

O padrão de distribuição da produtividade dos autores sobre o tema GECON também foi analisado. Fez-se a comparação com o mais famoso padrão de distribuição da produtividade dos autores, normalmente conhecido com Lei de Lotka ou Lei do Quadrado Inverso.

O índice proposto por Lotka (1926), que mensura o grau de produtividade dos autores, que será analisado nesse artigo, é calculado através da seguinte fórmula: $a_{n}=a_{1} / n^{c}$, onde an corresponde ao número de autores com $\mathrm{n}$ artigos; al corresponde ao número de autores que publicaram apenas um artigo; e $n$ corresponde ao número de artigos; e $c$ corresponde ao coeficiente de Lotka $(\approx 2)$.

Para se fazer a análise da distribuição da produtividade, foram descontadas a duplicidade de autoria dos autores que produziram artigos publicados tanto em evento quanto em periódico.

Tabela 6: Padrão de distribuição da produtividade dos autores sobre GECON e Padrão de Lotka

\begin{tabular}{lccc}
\hline Número de artigos por autor & Quantidade de autores & $\mathbf{( \% )}$ & Padrão de Lotka \\
\hline 1 artigo & 129 & $79,6 \%$ & $60,8 \%$ \\
2 artigos & 18 & $11,1 \%$ & $15,2 \%$ \\
3 artigos & 6 & $3,7 \%$ & $6,8 \%$ \\
4 artigos & 4 & $2,5 \%$ & $3,8 \%$ \\
5 artigos & 2 & $1,2 \%$ & $2,4 \%$ \\
6 artigos & 2 & $1,2 \%$ & $1,7 \%$ \\
7 artigos & 0 & $0,0 \%$ & $1,2 \%$ \\
Mais de 7 artigos & 1 & $0,6 \%$ & $8,1 \%$ \\
\hline Total & $\mathbf{1 6 2}$ & $\mathbf{1 0 0 , 0 \%}$ & $\mathbf{1 0 0 , 0 \%}$ \\
\hline
\end{tabular}

Fonte: Dados da pesquisa.

Foram necessários 162 autorias para a construção dos 90 artigos analisados (11 de periódicos e 79 de eventos). Dessa forma, identificou-se que a distribuição de autoria sobre GECON é aproximada da Lei de Lotka embora haja um percentual maior de autores $(79,6 \%)$, que publicou apenas um artigo sobre o tema, do que a Lei de Lotka previa $(60,8 \%)$. Além do que apenas 15 autores publicaram 3 ou mais artigos sobre o tema.

Na pesquisa de Passos et al. (2010) o número de autores que publicou apenas um trabalho foi de $75 \%$, ou seja, os autores também encontraram um volume maior de trabalhos sobre GECON publicados por autores que só produziram um trabalho do que a Lei de Lotka previa (que era de $60,8 \%$ ) e um resultado mais próximo daquele que esta pesquisa encontrou $(79,6 \%)$.

Os pesquisadores que concentram as autorias sobre o tema são:

Tabela 7: Produtividade dos autores em eventos e periódicos.

\begin{tabular}{|c|c|c|}
\hline Autor & Quantidade de artigos & (\%) sobre o total \\
\hline CATELLI, A. & 8 & $8,9 \%$ \\
\hline $\begin{array}{l}\text { PARISI, C.; } \\
\text { PONTE, V. M. R. }\end{array}$ & 6 & $6,7 \%$ \\
\hline $\begin{array}{l}\text { PEREIRA, C. A.; } \\
\text { SANTOS, E. S. }\end{array}$ & 5 & $5,6 \%$ \\
\hline $\begin{array}{l}\text { PETER, M. G. A.; } \\
\text { PESSOA, M. N. M.; } \\
\text { GUERREIRO, R.; } \\
\text { SANTOS, R. V. }\end{array}$ & 4 & $4,4 \%$ \\
\hline $\begin{array}{l}\text { OLIVEIRA, A. B. S.; } \\
\text { PETER, F. A.; }\end{array}$ & 3 & $3,3 \%$ \\
\hline
\end{tabular}

R. Cont. Ufba, Salvador-Ba, v. 9, n. 1, p. 41 - 57, jan-mar 2015 
TEIXEIRA, F. R. B.;

CROZATTI, J.;

LUSTOSA, P. R. B.;

PINHO, R. C. S.

Fonte: Dados da pesquisa.

Pode-se observar que o professor Armando Catelli foi o pesquisador que mais publicou artigos sobre o tema nos veículos analisados dentro desta bibliometria publicando 8 trabalhos o que corresponde a 8,9\% dos artigos publicados. Na sequência, aparecem os pesquisadores Parisi, C. e Ponte, V. M. R. com seis artigos cada seguidos de Peter, M. G. A., Pessoa, M. N. M., Guerreiro, R. e Santos, R. V. com quatro artigos cada.

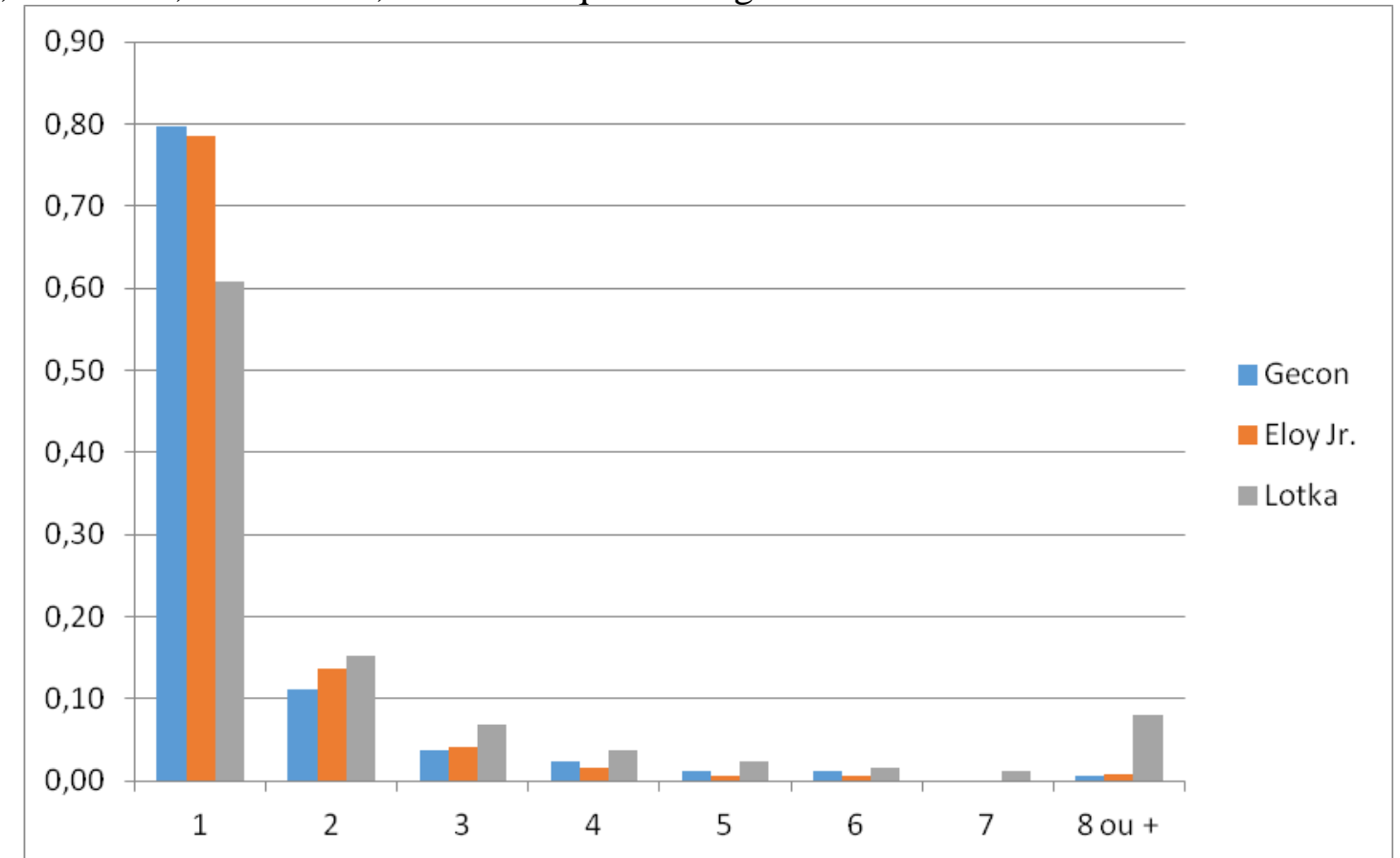

Figura 1: Distribuição da produtividade dos autores sobre GECON, segundo Eloy Jr., Soares e Casagrande (2013) e segundo a Lei de Lotka.

Fonte: Dados da Pesquisa.

Outro ponto interessante a se analisar é que a distribuição encontrada nesta pesquisa apresenta uma função mais próxima da pesquisa de Eloy Jr., Soares e Casagrande (2013) do que da Lei de Lotka.

Para analisar o impacto das publicações quanto ao número de citações, foi realizado uma análise pelo software Publish or Perish com posterior confirmação com o uso direto do Google Acadêmico, que resultou na Tabela 7, que demonstra o número de citações dos artigos em periódicos.

Tabela 8: Número de citações dos artigos de GECON publicados nos periódicos.

\begin{tabular}{|c|c|c|c|c|}
\hline Título & Periódico* & Citações & Ano & $\begin{array}{l}\text { Citações } \\
\text { por ano }\end{array}$ \\
\hline Gestão Econômica das Empresas na Nova Economia & CVR & 0 & 2001 & 0,00 \\
\hline Internet: Desafio para uma Contabilidade Interativa & $\mathrm{RCF}$ & 13 & 2001 & 1,08 \\
\hline $\begin{array}{l}\text { Uma Contribuição à Discussão Sobre a Avaliação de Desempenho } \\
\text { das Instituições Federais de Ensino Superior - Uma Abordagem da } \\
\text { Gestão Econômica }\end{array}$ & $\mathrm{RCF}$ & 13 & 2002 & 1,18 \\
\hline Gestão Econômica de Investimento em Ativos Fixos & $\mathrm{RCF}$ & 8 & 2003 & 0,80 \\
\hline
\end{tabular}

R. Cont. Ufba, Salvador-Ba, v. 9, n. 1, p. 41 - 57, jan-mar 2015 


\begin{tabular}{|c|c|c|c|c|}
\hline $\begin{array}{l}\text { Modelo de Apuração de Resultado de Redes de Varejo sob o } \\
\text { Enfoque da Gestão Econômica - GECON }\end{array}$ & $\mathrm{RCF}$ & 2 & 2003 & 0,20 \\
\hline $\begin{array}{l}\text { Uma Contribuição a Formulação de um Modelo de Apuração de } \\
\text { Resultados para Empresas de Carcinicultura sob a Ótica do } \\
\text { GECON }\end{array}$ & Contextus & 1 & 2004 & 0,11 \\
\hline Mensuração do Resultado & $\mathrm{RCC}$ & 0 & 2004 & 0,00 \\
\hline $\begin{array}{l}\text { Indicadores Financeiros e Não Financeiros de Longo Prazo: um } \\
\text { estudo de empresas atacadistas e distribuidoras brasileiras }\end{array}$ & CVR & 3 & 2006 & 0,43 \\
\hline $\begin{array}{l}\text { Gestão Economica em Instituições de Ensino Superior: } \\
\text { Mensuração de Resultados por Unidade de Negócios }\end{array}$ & $\mathrm{RCC}$ & 0 & 2007 & 0,00 \\
\hline As Respostas do GECON às Críticas do Relevance Lost & CVR & 0 & 2011 & 0,00 \\
\hline $\begin{array}{l}\text { Valor Econômico Gerado por Instituições Públicas: Avaliaçãa do } \\
\text { IFPA Campus Abaetetuba }\end{array}$ & CVR & 0 & 2012 & 0,00 \\
\hline
\end{tabular}

É possível verificar que apenas 4 dos 15 periódicos analisados veicularam artigos com a temática GECON. A Lei de Bradford, em bibliometria, permite identificar o grau de relevância que um determinado periódico, ou que um determinado grupo de periódicos assume na veiculação de artigos sobre determinados temas. Aqui, embora não se possa determinar efetivamente que a RCF, a CVR, a RCC e a Contextus, componham esse núcleo de periódicos devido à escolha intencional de análise feita pelos autores da amostra de periódicos que se limitou aos periódicos mantidos por programas de pós-graduação stricto sensu em controladoria e contabilidade, fica claro o indício de que estas 4 revistas, podem realmente compor esse núcleo previsto pela Lei de Lotka para o tema GECON.

No entanto, embora os 4 periódicos tenham veiculado artigos sobre GECON, aqueles veiculados pela RCF foram, de longe, mais citados que os veiculados pelas demais revistas, sendo que os 4 artigos veiculados pela RCF receberam um total de 36 citações, contra 3 citações de um artigo publicado na CVR e 1 citação do artigo da Contextus. Os 3 demais artigos da CVR e os dois da RCC não receberam nenhuma citação, o que pode indicar que, para ser citado, o trabalho deve preferencialmente ser publicado na RCF.

Finalizando a análise dos resultados encontrados, deve-se mencionar que há uma relação curiosa entre as autorias dos artigos de acordo com os autores mais produtivos. Tal relação, já encontrada por Soares e Vicente (2013) quando estes analisaram os autores de livros sobre Teoria da Contabilidade, é uma relação de orientação em programas de pós-graduação.

O autor Catteli, A. foi orientador dos autores: Parisi, C. (no MSc e PhD), Ponte, V. M. R. (no $\mathrm{PhD}$ ), Pereira, C. A. (no MSc e PhD), Guerreiro, R. (no MSc e PhD) e Oliveira, A. B. S. (no $\mathrm{MSc}$ e $\mathrm{PhD}$ ), todos pelo PPGCC/USP.

$\mathrm{O}$ autor Guerreiro, R. orientou a autora Santos, E. S. (MSc e PhD), o autor Santos, R. V. (MSc) e o autor Crozatti, J. (PhD) também pelo PPGCC/USP.

Os autores Crozatti, J., Pinho, R. C. S. e Peter, M. G. A. concluíram seus mestrados e os autores Santos, R. V. e Lustosa, P. R. B. concluíram seu doutorados também pelo PPGCC/USP.

A autora Pessoa, M. N. M fez seu mestrado em economia, os autor Peter, F. A. e Teixeira, F. R. B fizeram seus mestrados profissionais em controladoria, e as autoras Peter, M. G. A. e Pinho, R. C. S fizeram seus doutorados em educação, todos pela UFC.

Parte desta relação de ligações entre os autores por meio de orientações já havia sido detectada no trabalho de Kroenke, Söthe e Bezerra (2011) embora estes não tivessem detectado que a relação incluiria além das orientações, um tema em comum.

R. Cont. Ufba, Salvador-Ba, v. 9, n. 1, p. 41 - 57, jan-mar 2015 
O autor Catelli, A. foi um dos professores que mais orientou trabalhos dentro do PPGCC/USP totalizando 57 mestres e doutores. Para fins de comparação, o professor Martins, E. orientou 54, o professor Santos, A. orientou 49 e o professor Carvalho, L. N. G. orientou 42 (dados consultados no mês de agosto de 2013).

De fato, vale lembrar a síntese de Meadows sobre o assunto (1999, p. 93), “[...] verifica-se com frequência que eminentes pesquisadores de hoje em dia foram ensinados por eminentes pesquisadores da geração anterior”.

\section{CONSIDERAÇÕES FINAIS}

A pesquisa bibliométrica teve como objetivo verificar os trabalhos publicados em periódicos e eventos que abordaram o tema de gestão econômica no período de 1989 a 2012. Após a amostra ser selecionada, os artigos foram analisados cuidadosamente e foram retiradas as principais características que servem de interesse a esta pesquisa. A intenção deste trabalho foi ampliar os estudos anteriores existentes no que tange ao número de anos e a diversidade de periódicos e eventos analisados, para que, de alguma forma, propicie a real evolução da pesquisa sobre o tema.

O primeiro ponto a ser destacado é que dos 15 periódicos analisados (ligados a programas de pós-graduação stricto sensu em controladoria e contabilidade) apenas 4 veicularam artigos sobre o tema. Por um lado há duas justificativas para ambos: i. para que um artigo seja aceito em um periódico faz-se necessário que tal periódico disponha de avaliadores que dominem o assunto e ii. e Lei de Bradford explica que um pequeno grupo de periódicos pode, naturalmente, tornar-se o veículos de publicação de artigos sobre determinado tema.

Já quanto aos eventos, também houve a ocorrência daqueles que não veicularam nenhum artigo sobre o tema (EnEPQ e AnpCont) e uma forte concentração de veiculação no Congresso Brasileiro de Custos. Uma das explicações possíveis para isso, além das aplicáveis aos periódicos, é que os dois eventos tiveram suas primeiras edições (Anpcont e EnEPQ em 2007) posteriormente ao período de maior produtividade conforme indicado nesta pesquisa e na pesquisa de Carvalho et al. (2010).

Os materiais consultados pelos artigos publicados em eventos e periódicos diferiram levemente em qualidade sendo que as consultas dos artigos de periódicos se basearam menos em livros e monografias, e mais em teses e artigos de eventos e de outros periódicos. O próprio número médio de referências também foi maior em artigos de periódicos $(24,4)$ do que em eventos $(17,5)$ o que pode ser um indício de busca de melhorias nas versões definitivas.

O número de autores por artigo em eventos foi de 2,57 e em periódicos foi de 2,27. Em eventos a amplitude foi de 1 a 6 autores e em periódicos foi de 1 a 4 . Tal distribuição é natural dado que é sabido que os eventos como o EnAnpad aceitam até seis autores e periódicos costumem aceitar até 4 autores.

A análise proposta nesta pesquisa pretendeu identificar a distribuição da produção dos artigos e baseou-se na Lei de Lotka para isto. O que foi possível identificar é que sobre o tema GECON, há um percentual maior de autores que faz só um artigo $(79,6 \%)$ do que a prevista pela Lei de Lotka $(60,8 \%)$. Por outro lado, a concentração de autores que publicou mais de sete artigos sobre GECON foi muito menor $(0,6 \%)$ do que a Lei de Lotka previa $(8,1 \%)$. A produtividade dos autores sobre GECON é mais parecida com a produtividade de autores sobre contabilidade tributária analisada na pesquisa de Eloy Jr., Soares e Casagrande (2013) do que da Lei de Lotka.

O grupo autores que publicou mais de 3 artigos é composto por quinze pesquisadores entre os quais foi possível identificar uma forte relação por meio de orientação acadêmica no

R. Cont. Ufba, Salvador-Ba, v. 9, n. 1, p. 41 - 57, jan-mar 2015 
desenvolvimento de teses e e dissertações e por meio de localização institucional. O autor Catelli, A. orientou outros cinco dos pesquisadores mais produtivos, incluindo o autor Guerreiro, R. que por sua vez orientou outros 3 autores mais produtivos. O vínculo institucional destes autores foi grandemente relacionado ao PPGCC/USP e a UFC.

Foi também possível identificar que artigos publicados através da Revista de Contabilidade e Finanças foram muito mais citados que os artigos citados pelas revistas Contabilidade Vista e Revista, Revista Contemporânea de Contabilidade e Contextus, o que vai ao encontro do fator de impacto calculado para estas revistas por Souza, Ensslin e Ensslin (2012).

A análise realizada nesta pesquisa permitiu ainda identificar que inúmeros trabalhos trataram da adequação do GECON em empresas de diversos setores como bancário, hospitalar, setor público, setor de serviços, cooperativas e pequenas empresas o que mostra que pode haver um certo esgotamento desta abordagem nas pesquisas. A lacuna que falta ser preenchida envolve a investigação dos modelos de decisão dos gestores e como se pode tornar o GECON mais aplicável por meio de sua simplificação. Este é um dos caminhos de pesquisa que pode ser percorrido pelos pesquisadores de GECON.

Por fim, pode-se indicar várias sugestões para trabalhos futuros baseado nas lacunas que esta pesquisa identificou como, identificar com base nos currículos Lattes dos pesquisadores mais produtivos, se estes se utilizam de outras revistas que não as desta amostra para veicularem seus artigos sobre GECON e também tentar explicar os fatores que trouxeram a pesquisa sobre GECON ao estado da arte que ela se encontra hoje.

\section{REFERÊNCIAS}

CAPES. Ministério da Educação. Comunicado n. 002/2012, de 04 de maio de 2012. Atualização do Webqualis da Área. Brasília. Disponível em : http://www.capes.gov.br/images/stories/download/avaliacao/Qualis_Administracao.pdf Acesso em: 15 jul. 2013.

CAPES. Ministério da Educação. Documento de Área - 2007/2009, de $1^{\circ}$ de dezembro de 2009. Brasília. Disponível em

:http://www.capes.gov.br/images/stories/download/avaliacao/ADMIN17jun10.pdf Acesso em: 15 jul. 2013.

CARVALHO, F. S.; SALES, I. C. H.; MIRANDA, R. F. A.; SOUZA, L. M. S. Gecon: Uma Análise das Metodologias e Técnicas de Pesquisa dos Trabalhos Publicados Entre 1989 e 2010. In: XVII Congresso Brasileiro de Custos. Belo Horizonte. Anais... Belo Horizonte, MG, 2010.

CATELLI, A.; GUERREIRO, R.; PEREIRA, C. A. Avaliação de Resultados e Desempenhos em Instituições Financeiras. In: CATELLI, A. (Coord.). Controladoria: uma abordagem da gestão econômica - GECON. 2 ed. São Paulo: Atlas, 2010. Cap. 13, p. 359-385.

CATELLI, A.; GUERREIRO, R.; PEREIRA, C. A.; ALMEIDA, L. B. Sistema de Gestão Econômica - GECON. In: CATELLI, A. (Coord.). Controladoria: uma abordagem da gestão econômica - GECON. 2 ed. São Paulo: Atlas, 2010. Cap. 9, p. 285-306.

CRUZ, A. P. C.; MACHADO, E. A.; MARTINS, G. A.; ROCHA, W. Da Pesquisa em Construção à Publicação Definitiva Conversão da Produção Científica no Campo da 
Contabilidade (2001-2010). In: CONGRESSO USP DE CONTROLADORIA E

CONTABILIDADE, 11, 2011, São Paulo. Anais... São Paulo: FEA/USP, 2011.

CRUZ, A.P.C.; PEREIRA, C.A.; ESPEJO, M.M.S.B. Análise retrospectiva do debate sobre gestão econômica. In: XVIII Congresso Brasileiro de Custos - Rio de Janeiro, RJ, Brasil.

Anais... Rio de Janeiro, RJ, Brasil, 07 a 09 de novembro de 2011.

ELOY JUNIOR, A. C. C.; SOARES, S. V.; CASAGRANDE, M. D. H. A produção científica brasileira sobre Contabilidade Tributária em periódicos e eventos no período de 1989-2011.

Revista de Contabilidade e Controladoria. No prelo. 2014.

GUERREIRO, R. Modelo conceitual de sistema de informação de gestão econômica: uma contribuição à teoria da comunicação da contabilidade. São Paulo, 1989. Tese (Doutorado) Faculdade de Economia, Administração e Contabilidade, Universidade de São Paulo.

GUERREIRO, R.; CATELLI, A.; DORNELLES, J. A. A Controladoria sob o enfoque Gecon - Gestão Econômica: A Experiência da Caixa Econômica Federal do Brasil. Revista de Contabilidade do CRC-SP, São Paulo, jul., p. 19-25, 1997.

KROENKE, A.; SÖTHE, A.; BEZERRA, F. A. Evolução do conhecimento contábil nos artigos científicos da revista de contabilidade e finanças sob a visão epistemológica de Ludwik Fleck. Enfoque: Reflexão Contábil, v. 30., n. 2, p. 83-97 maio/ago., 2011.

MEADOWS, A. J. A Comunicação Científica. Brasília: Briquet de Lemos, 1999.

MURCIA, F. C. S.; ROSA, C. A. ; BORBA, J. A. . Produção Científica em Ciências Contábeis: uma comparação entre a meta estabelecida pela CAPES e a publicação de artigos por parte dos docentes de Programas de Pós-Graduação. Contabilidade, Gestão e Governança, v. 16, p. 68-81, 2013.

PARISI, C.; CORNACHIONE JÚNIOR, E. B.; VASCONCELOS, M. T. de C. Modelos de Identificação e acumulação de Resultados. In: CATELLI, A. (Coord.). Controladoria: uma abordagem da gestão econômica - GECON. 2 ed. São Paulo: Atlas, 2010. Cap. 13, p. 323339.

PASSOS, G.R.P.; ROS, R.; MEGLIORINI,E.;PARISI,C. Inserção do modelo de gestão econômica - GECON em periódicos científicos nacionais com classificação QUALIS A ou B: uma análise bibliométrica no período de 1993 a 2008. In:XVII Congresso Brasileiro de Custos. Belo Horizonte. Anais... Belo Horizonte, MG, 2010.

PEREIRA, C. A. Gestão Econômica. IN: PARISI, C. e MEGLIORINI, E. (Organiz.). Contabilidade Gerencial. São Paulo, Atlas, 2011. Cap. 9, p. 191-238.

RIBEIRO, S. P.; TISOTT, S. T.; TONELLO, D.; MAGNI, A. A. Estudo bibliométrico em controladoria no congresso brasileiro de custos no período de 2004 a 2010. In: XVIII Congresso Brasileiro de Custos. Rio de Janeiro. Anais... Rio de Janeiro, RJ, Brasil, 2011.

SALES, I. C. H.; MIRANDA, R. F. A.; CARVALHO, F. S.; SOUZA, L. M.; SANTANA, C. M. 22 Anos de Pesquisas Contábeis sobre GECON. Revista Ciências Sociais em Perspectiva. Cascavél. Vol. 10, n.19, 2011. 
SOARES, S. V. ; VICENTE, E. F. R. . Bibliografia brasileira sobre Teoria da Contabilidade: o que se encontra nos livros?. In: X Congresso Online de Administração, 2013, online. Anais, 2013.

SOARES, Sandro Vieira et al. Evolução do currículo de Contabilidade no Brasil desde 1809. Revista Catarinense da Ciência Contábil, v. 10, n. 30, p. 27-42, 2012.

SOUZA, J. V. ; ENSSLIN, S. R. ; ENSSLIN, L. Panorama dos Periódicos vinculados à área contábil quanto ao reconhecimento da Sociedade Científica. In: Congresso USP de Controladoria e Contabilidade, 2012, São Paulo. 12 Congresso USP de Controladoria e Contabilidade, 2012. 